\title{
Wideband Bandpass Filter with Excellent Selectivity Using Anti-coupled Line
}

\author{
Liming Liang ${ }^{1,2, a}$,Shulan $\mathrm{Li}^{1,2, \mathrm{~b}}$, Yuanan Liu ${ }^{1,2, \mathrm{c}}$, Cuiping $\mathrm{Yu}^{1,2, \mathrm{~d}}$ \\ ${ }^{1}$ Beijing Key Laboratory of Work Safety Intelligent Monitoring, \\ Beijing University of Posts and Telecommunications, \\ Beijing, China \\ ${ }^{2}$ School of Electronic Engineering, \\ Beijing University of Posts and Telecommunications, \\ Beijing, China \\ ae-mail: liang.19841220@163.com, be-mail: lishulan@bupt.edu.cn, \\ ce-mail: yuliu@bupt.edu.cn, ${ }^{\mathrm{d} e-m a i l: y u c u i p i n g @ b u p t . e d u . c n, ~}$
}

\begin{abstract}
A simple anti-coupled line circuit configuration is proposed to design wideband bandpass filter with sharp skirt selectivity in this paper. Good skirt selectivity can be achieved with two transmission zeros near the passband. The location of transmission zeros and bandwidth of passband can be adjusted by changing the impedance of the configuration. A compact wideband bandpass filter prototype with $20 \mathrm{~dB}$ rejection bandwidth of $61.4 \%$ is designed and fabricated. In order to further improve the skirt selectivity and stopband rejection level, two identical filter prototypes were directly cascaded. The cascaded filter exhibits a competitive attenuation slope of 262.2 and $75.9 \mathrm{~dB} / \mathrm{GHz}$ in the lower and upper passband transitions, respectively.
\end{abstract}

Keywords-anti-coupled line; bandpass filter; transmission zeros; rejection bandwidth; skirt selectivity

\section{INTRODUCTION}

Bandpass filters have been applied to many microwave and millimeter communication systems. They are commonly used to separate different frequencies. With the rapid development of modern wireless wideband systems, low-cost high-performance wideband bandpass filters become very necessary. Recently, much research work on wideband bandpass filter has been performed. Various wideband bandpass filters employing different methods have been designed and analyzed [1-3]. A wideband bandpass filter with $3 \mathrm{~dB}$ fractional bandwidth of $62.7 \%$ using a symmetric multimode resonator is implemented in [1], and a wideband bandpass filter with excellent selectivity adopting complementary split-ring resonators (CSRRs) resonator is presented in [2]. In [3], a low insertion loss wideband bandpass filter with six transmission zeros based on opencircuited transmission-line resonator is introduced.

Anti-coupled line, as a new circuit configuration, has been proposed to design compact and wideband bandstop filter [4], low-pass filters [5], wilkinson power divider [6], and bandpass filters [7]. The obvious feature of anti-coupled line is inherently simple circuit while its external performance can satisfy flexible requirements by choosing special electrical parameters, as discussed in [4-7]. In this paper, by introducing additional terminated coupled-line section connected with two symmetrical open-circuited transmission-line stubs into anti-coupled line, a novel coupled-line circuit configuration is proposed for wideband bandpass filter applications. By changing the characteristic impedance of these coupled-lines and transmission-line stubs, the bandwidth of the passband can be controlled easily. In addition, two transmission zeros near the passband can be achieved to improve the selectivity of the wideband filter. A prototype of the wideband bandpass filter operating at $2.8 \mathrm{GHz}$ with a broad lower stopband $(|\mathrm{S} 21|>20 \mathrm{~dB}$, from 0.91 to $1.89 \mathrm{GHz}$ ) has been designed. The detailed theoretical design, and the simulation and experimental results are demonstrated and discussed.

\section{The Circuit Structure AND TheORY OF THE PROPOSED BANDPASS FILTER}

Fig. 1 shows the proposed anti-coupled line structure to design novel wideband bandpass filters. This circuit configuration is composed of two-section coupled lines with a common connected point $\mathrm{A}$, and two symmetrical opencircuited transmission-line stubs. Where, Z1e, Z1o, Z2e, Z2o, $\mathrm{Z} 3, \theta 1, \theta 2$ and $\theta 3$ represent the characteristic impedances and the electrical lengths of coupled lines and open-circuited transmission-line stubs, respectively. According to the analysis method for symmetrical network, this circuit analysis can be bisected into two identical halves with respect to its symmetrical interface. When an even-mode excitation is used, the total circuit configuration shown in Fig. 1 becomes two identical one-port networks as indicated in Fig. 2(a). Similarly, the one-port and odd-mode network under an odd-mode excitation shown in Fig. 2(b) can be obtained. 


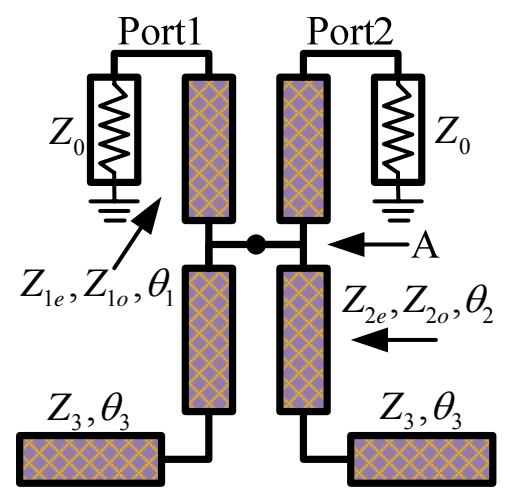

Figure 1. The proposed anti-coupled line structure for bandpass filter prototype.

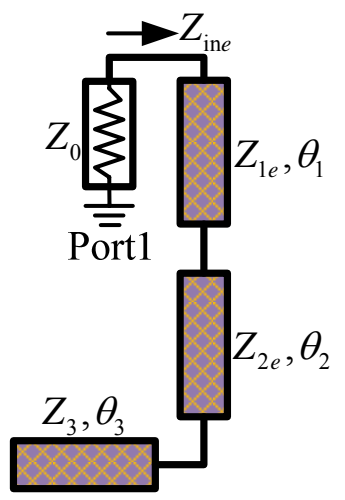

(a)

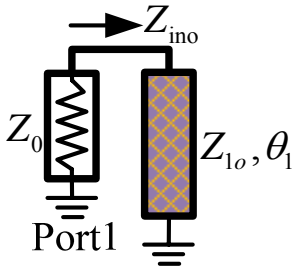

(b)
Figure 2. The equivalent simplified circuit structures of the bandpass filter prototype. (a) even-mode analysis. (b) odd-mode analysis.

If the one-port, even- and odd-mode networks' scattering parameters S11e and S11o are determined, the two-port scattering parameters can be expressed by:

$$
S_{11}=\frac{S_{11 e}+S_{11 o}}{2} \quad S_{21}=\frac{S_{11 e}-S_{110}}{2}
$$

Furthermore, the input impedances Zine and Zino indicated in Fig. 2(a) and Fig. 2(b) can be calculated by:

$$
Z_{\text {ine }}=\frac{j Z_{1 e}\left(\begin{array}{l}
Z_{1 e} Z_{3} \tan \theta_{1} \tan \theta_{2}+Z_{1 e} Z_{2 e} \tan \theta_{1} \tan \theta_{3} \\
+Z_{2 e} Z_{2 e} \tan \theta_{2} \tan \theta_{3}-Z_{2 e} Z_{3}
\end{array}\right)}{\left(\begin{array}{l}
Z_{2 e} Z_{3} \tan \theta_{1}+Z_{1 e} Z_{3} \tan \theta_{2}+Z_{1 e} Z_{2 e} \tan \theta_{3} \\
-Z_{2 e} Z_{2 e} \tan \theta_{1} \tan \theta_{2} \tan \theta_{3}
\end{array}\right)}
$$

Thus, the reflection coefficients S11e and S11o for Fig. 2(a) and Fig. 2(b) can be described by:

$$
S_{11 k}=\frac{Z_{i n k}-Z_{0}}{Z_{i n k}+Z_{0}}, k=o, e .
$$

By substituting (2), (3) and (4) into (1), the final mathematical expression of scattering parameters S11 and S22 for Fig. 1 can be obtained as:
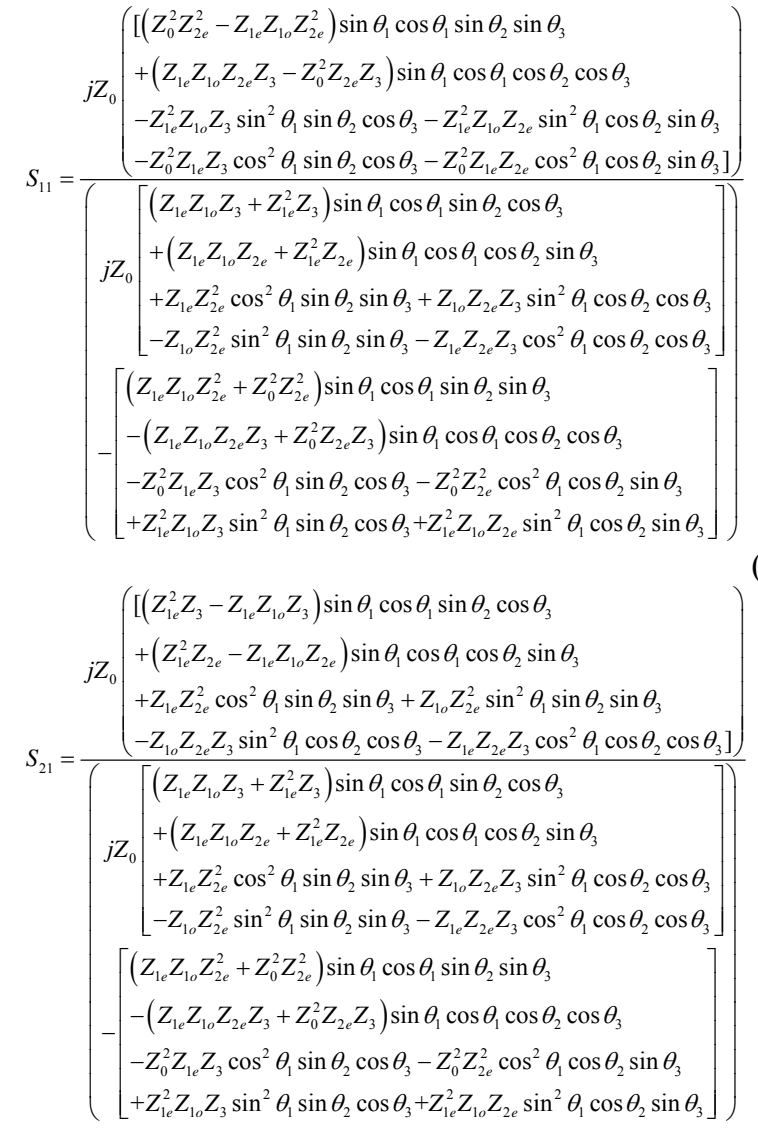

In general, the scattering parameters of bandpass filter should satisfy the conditions: $|\mathrm{S} 11|=0$ and $|\mathrm{S} 21|=1$ for ideal in-band performance. Here, we assume that the center frequency of this novel bandpass filter is $2.8 \mathrm{GHz}$, and the values of the electrical lengths $\theta 1, \theta 2$ and $\theta 3$ correspond to $2.8 \mathrm{GHz}$. When $|\mathrm{S} 11|=0$ and $|\mathrm{S} 21|=1$ are considered, respectively, we can obtain the design electrical lengths $\theta 1=$ $\Pi$ and $\theta 2=\theta 3=\Pi / 2$.

\section{Simulated AND MEASURED RESUltS}

The proposed filter prototype is implemented on a microstrip substrate with Rogers R04350B with relative dielectric constant of 3.48 and thickness of $0.762 \mathrm{~mm}$. A commercial full-wave simulator, ANSOFT HFSS 14, is utilized for further analysis and optimization. For a BPF with $20 \mathrm{~dB}$ fractional bandwidth of $63 \%$ and center frequency of $2.8 \mathrm{GHz}$, the optimized physical dimension values shown in Fig. 3 are: $\mathrm{L} 0=20.8 \mathrm{~mm}$ ( $50 \mathrm{Ohm}$ microstrip line of input and output ports ), W0 $=1.69 \mathrm{~mm}, \mathrm{~L} 1=35 \mathrm{~mm}, \mathrm{~W} 1=0.34$ $\mathrm{mm}, \mathrm{S} 1=0.26 \mathrm{~mm}, \mathrm{~L} 2=15.4 \mathrm{~mm}, \mathrm{~W} 2=0.56 \mathrm{~mm}, \mathrm{~S} 2=$ $0.41 \mathrm{~mm}, \mathrm{~L} 3=16.1 \mathrm{~mm}, \mathrm{~W} 3=0.3 \mathrm{~mm}$. Fig. 4(a) and Fig. 4(b) show the practical photograph of the final fabricated bandpass filter prototype and cascaded filter, respectively.

Fig. 5 demonstrates the simulated and measured results of bandpass filter prototype where good agreement can be observed. The filter prototype exhibits $20 \mathrm{~dB}$ fractional bandwidth of approximately $61.4 \%(1.89-3.61 \mathrm{GHz})$ with 
the insertion and return losses less than $1.2 \mathrm{~dB}$ and better than $12.5 \mathrm{~dB}$ over the entire passband, respectively. In addition, the measured group delay from 2.15 to $3.4 \mathrm{GHz}$ is between $0.13-2.0 \mathrm{~ns}$. In order to further improve the skirt selectivity and stopband rejection level, two identical filter prototypes were directly cascaded, as shown in Fig. 4(b). Fig. 6 illustrates the simulated and measured performances of the cascaded filter. The stopband attenuation of more than $40 \mathrm{~dB}$ on the left side of the passband $(0.91-1.84 \mathrm{GHz})$, and also at the right side $(3.62-4.7 \mathrm{GHz})$ is achieved. Specifically, the implemented cascaded filter exhibits a competitive attenuation slope with 262.2 and $75.9 \mathrm{~dB} / \mathrm{GHz}$ in the lower and upper passband edges, respectively.

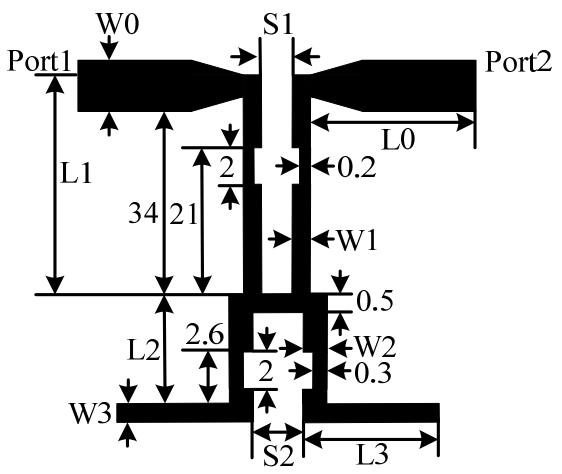

Figure 3. Layout of proposed bandpass filter prototype.

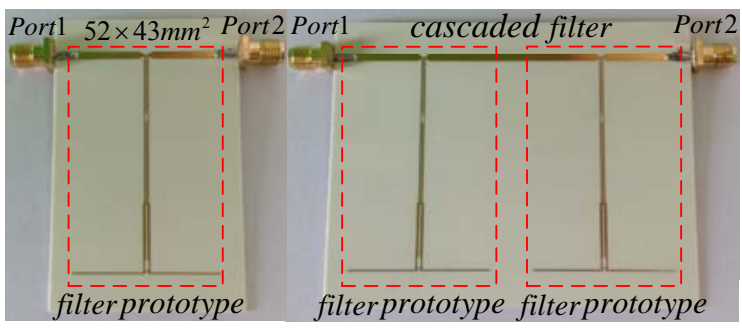

(a)

(b)

Figure 4. Photographs of the fabricated filter. (a) filter prototype. (b) cascaded filter.

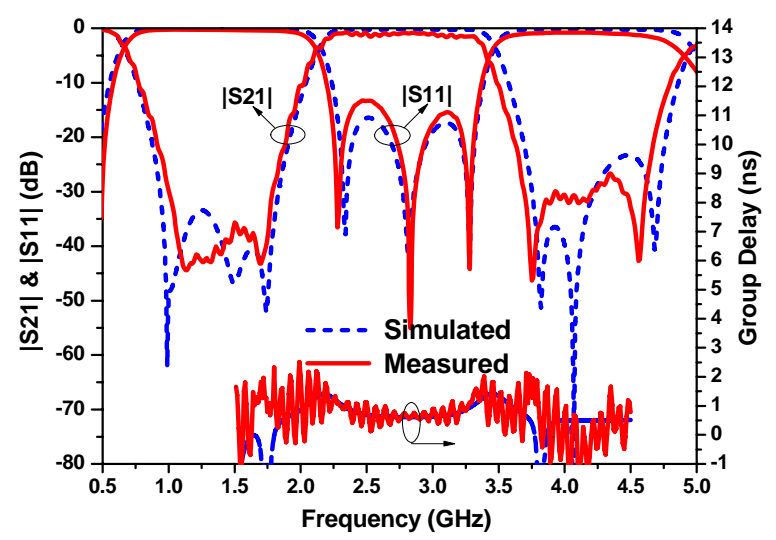

Figure 5. Simulated and measured results of the filter prototype.

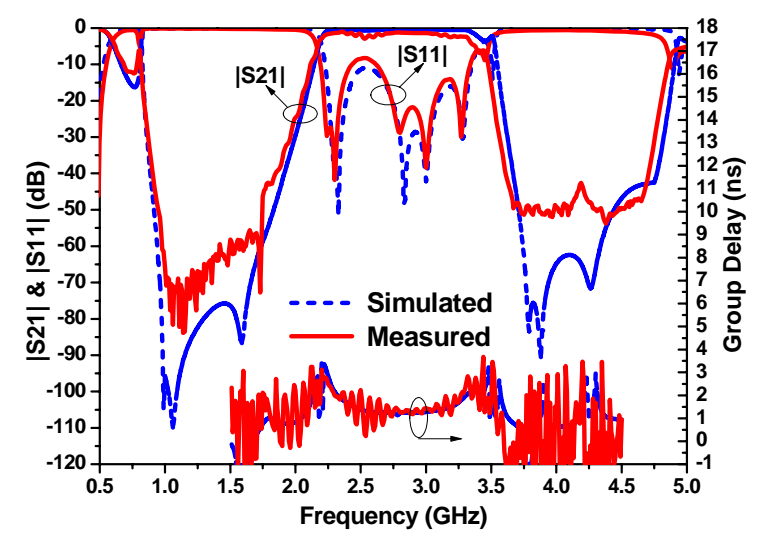

Figure 6. Simulated and measured results of the cascaded filter.

\section{SUMMARY}

In this paper, a novel wideband bandpass filter is realized using anti-coupled line circuit structure. A compact prototype filter with $61.4 \%$ rejection bandwidth is demonstrated. In order to further improve the skirt selectivity and stopband rejection level, two identical filter prototypes were directly cascaded. The cascaded filter exhibits a competitive attenuation slope of 262.2 and $75.9 \mathrm{~dB} / \mathrm{GHz}$ in the lower and upper passband transitions, respectively.

\section{ACKNOWLEDGEMENT}

This work was supported in part by National Natural Science Foundation of China (No.61201025, No.61327806), and Fundamental Research Funds for the Central Universities (No. 2013RC0204).

\section{REFERENCES}

[1] W.-J. Feng, J.-G. Zhou, and W.-Q. Che, "Wideband bandpass filter using symmetric multimode resonator," Electron. Lett., 2012, 48, (17), pp. 1071-1073

[2] X. Luo, H. Qian, J.-G. Ma, and E.-P. Li, "Wideband bandpass filter with excellent selectivity using new CSRR-based resonator," Electron. Lett., 2010, 46, (20), pp. 1390 -1391

[3] F.-C. Chen, J.-M. Qiu, Z.-H. Chen, and Q.-X. Chu, "Low insertion loss wideband bandpass filter with six transmission zeros," Electron. Lett., 2013, 49, (7), pp. $477-479$

[4] M.-Y. Hsieh, and S.-M. Wang, "Compact and wideband microstrip bandstop filter," IEEE Microwave Wireless Compon. Lett., 2005, 15, (7), pp. 472-474

[5] R. Li, D.-I. Kim, and C.-M. Choi, "Compact structure with three attenuation poles for improving stopband characteristics," IEEE Microwave Wireless Compon . Lett., 2006, 16, (12), pp. 663-665

[6] J. Zhang, L. Li, J. Gu, and X. Sun, "Compact and harmonic suppression wilkinson power divider with short circuit anti-coupled line," IEEE Microwave Wireless Compon . Lett., 2007, 17, (9), pp. 661-663

[7] C.-P. Chiang, and K.-W. Tam, "Compact quasi-elliptic microstrip bandpass filter using terminated anti-parallel coupled-line," IET Microwaves, Antennas \& Propagation, 2009, 3, (8), pp. 1206-1210 